\title{
Modeling and diagnostics for plasma discharge capillaries
}

\author{
A. Curcio \\ CERN, Geneva, Switzerland \\ F. Bisesto ${ }^{\bullet}$, G. Costa, A. Biagioni, M. P. Anania, R. Pompili $\odot$, and M. Ferrario \\ INFN LNF, Frascati (Rome), Italy \\ M. Petrarca \\ S.B.A.I. Department of the Roma University “La Sapienza," Rome, Italy \\ and INFN Roma1, Rome, Italy
}

(Received 31 July 2019; revised manuscript received 27 September 2019; published 18 November 2019)

\begin{abstract}
In this paper, we show how plasma discharge capillaries can be numerically modeled as resistors within an RLC-series discharge circuit, allowing for a simple description of these systems, while taking into account heat and radiation losses. An analytic radial model is also provided and compared to the numerical model for plasma discharge capillaries at thermal equilibrium, with corrections due to radiation losses. Finally, diagnostic techniques based on visible spectroscopy of plasma emission lines are discussed both for atomic and molecular gases, comparing experimental results with numerical simulations and theoretical calculations.
\end{abstract}

DOI: 10.1103/PhysRevE.100.053202

\section{INTRODUCTION}

Experiments on laser wakefield acceleration (LWFA) [1], plasma wakefield acceleration (PWFA) [2], and plasma lensing [3-7] have recently undergone a great deal of development. Because of the high gradients that plasma environments can sustain, accelerator physicists have been paying increasing attention to the field of plasma acceleration, conceiving novel accelerator designs and acceleration schemes [8] with the goal to provide the best performances in terms of beam emittance $[6,7,9]$, high energy-gain, and strong focusing [4,9]. Plasmas can sustain high gradients according to their electron plasma density. A common scheme is to confine plasmas within dielectric capillaries in order to enclose them into a fixed volume of space, thus controlling the plasma density [10-12]. Plasma production in capillary structures is the topic of interest of this paper. In particular, the focus is on plasmas generated by electric discharges in gases embedded within dielectric capillaries. For regimes of low plasma excitation, the following formula holds, which relates the maximum sustainable electric field $E_{\max }$ to the electron plasma density $n_{e}: E_{\max } \sim 96\left(n_{e}\left[\mathrm{~cm}^{-3}\right]\right)^{1 / 2} \mathrm{G} \mathrm{V} / \mathrm{m}$ [1]. For plasmas generated by electric discharges, the electron plasma density is a function of the electron temperature, since the degree of gasionization is firmly connected to plasma heating. Therefore, in this case the unique parameter describing a plasma discharge capillary is the electron temperature. This has already been widely discussed throughout local magnetohydrodynamics

Published by the American Physical Society under the terms of the Creative Commons Attribution 4.0 International license. Further distribution of this work must maintain attribution to the author(s) and the published article's title, journal citation, and DOI. numerical models [13]. Also analytic models at the equilibrium condition have been proposed and exploited for the interpretation of experimental data $[6,13]$. Here we propose a macroscopic description of the plasma discharge capillary, in the form of a numerical code, aiming to describe all the quantities of interest, such as the electron temperature, the electron plasma density, and the plasma discharge current. All the quantities are considered as averages over the plasma volume. Our description is not able to give local values of the plasma current density, of the associated magnetic field [14], and of the plasma density, but it can efficiently take into account the plasma heating, the heating of the capillary walls, i.e., the heat flux moving from the plasma to the walls of its container, and finally all the radiative losses due to bremsstrahlung radiation (BR) and the radiative recombination (RR). In this sense, the presented macroscopic model consists in an improvement of a preexisting one $[15,16]$, where many of these physical mechanisms were not included. Jointly, we propose an analytic model for the study of plasma discharge capillaries at thermal equilibrium, stressing the relevance of the radiation losses to the transverse profile of the plasma temperature and all the related quantities. Finally, experimental measurements of electron plasma density [17] through the spectroscopy of Stark-broadened emission lines are shown, compared to simulations obtained with the numerical code and also to theoretical calculations based on an analytic model specially developed for the atomic and molecular Stark-broadening in plasmas.

\section{GENERAL DESCRIPTION OF THE MODEL}

The model we are going to describe in this section is based on modeling the discharge circuit as an RLC-series, where the plasma is treated as a resistor. Power dissipated into the 
plasma resistor can flow into different processes during the time evolution of the discharge. These processes include gas ionization, plasma heating, and overall losses, encompassing heat and radiation losses. We are going to couple the equation of the RLC-series circuit with the energy conservation equation. The standard equation for the current flowing in the RLC-series circuit is

$$
\frac{d^{2} I_{p}}{d t^{2}}+\left(\frac{R+R_{p}}{L}\right) \frac{d I_{p}}{d t}+\left(\frac{1}{L C}\right) I_{p}=0,
$$

where $R$ is the parasitic resistance of the circuit (and $R_{p}$ is the plasma resistance), and $(L C)^{-1 / 2}$ is the circuit resonance frequency, with $L$ the parasitic inductance of the circuit and $C$ the electric capacitance charged, for example, by a thyratron. The solution for the current temporal profile will therefore be a capacitor discharge over the plasma resistance that varies in time. The energy conservation equation states that, at each instant, energy is "flowing in" due to Ohmic plasma heating and "flowing out" due to the heat and radiation losses. Therefore, the equation for the temperature of the resistor, i.e., the plasma temperature, will be [18]

$$
C_{V} \frac{d T}{d t}=R_{p} I_{p}^{2}-H_{w}-P_{\mathrm{RR}}-P_{\mathrm{BR}},
$$

where $H_{w}=H_{w e}+H_{w i}$ is the heat rate to the wall, encompassing both the electron $H_{w e}$ and ion $H_{w i}$ contributions, $P_{\mathrm{RR}}$ is the power flowing away through radiative recombination, and $P_{\mathrm{BR}}$ is the power flowing away because of bremsstrahlung radiation of plasma electrons colliding with plasma ions. When identifying the temperature that dissipated in the plasma resistor as the plasma temperature, a single-temperature plasma is understood, where the electron-ion collisions occur at a rate high enough the thermalize the two fluids (electron and ion fluids) in a time interval much shorter than the discharge timescale. Indeed, in this paper we consider discharge pulses as long as a few hundred nanoseconds, plasma electron temperatures of the order of a few eV, and plasma densities of the order of $10^{17}-10^{18} \mathrm{~cm}^{-3}$, corresponding to thermalization times of a few picoseconds. The common notation $k_{B}$ for the Boltzmann constant has been adopted. The heat capacity at a constant volume can be expressed as

$$
C_{V}=\frac{3}{2} \frac{P_{0} V}{T_{0}},
$$

where $P_{0}$ is the input gas pressure and $T_{0}$ is the initial temperature (room temperature). The plasma volume is $V=$ $\pi R_{\text {cap }}^{2} L_{\text {cap }}$, where we also have introduced the capillary radius and length, $R_{\text {cap }}$ and $L_{\text {cap }}$, respectively.

\section{A. Heat rate}

A deeper insight into the heat rate, defined as the heat flux out of the capillary surface $S_{\text {cap }}=2 \pi R_{\text {cap }} L_{\text {cap }}$, gives

$$
H_{w}=H_{w e}+H_{w i}=\iint_{S_{\text {cap }}}\left[\kappa_{e}(T)+\kappa_{i}(T)\right] \vec{\nabla} T \cdot \vec{d} S,
$$

where the electron and the ion thermal conductivities $\kappa_{(e, i)}$ are expressed through the Wiedemann-Franz law in terms of the electric conductivity $\sigma_{(e, i)}$, namely $\kappa_{e, i}=\left(\pi^{2} k_{B}^{2} / 3 e^{2}\right) \sigma_{e, i} T$. In turn, the electric conductivity is obtained from the Drude model applied both to the electron and the ion fluids. In the formula

$$
\sigma_{(e, i)}=Z_{\mathrm{eff}} \varepsilon_{0} \frac{\omega_{p,(e, i)}^{2}}{v_{(e i, i e)}},
$$

the electron or ion plasma frequency $\omega_{p,(e, i)}$ has been introduced as well as the electron-ion or ion-electron collision frequency $v_{(e i, i e)}$ [19]. The parameter $Z_{\text {eff }}$, discussed later in detail, is the effective degree of ionization of the gas (therefore the ionic charge), taking into account the multiionization of the gas atoms. The ratio of the electron to the ion conductivity is

$$
\frac{\sigma_{e}}{\sigma_{i}}=\frac{\kappa_{e}}{\kappa_{i}}=Z_{\mathrm{eff}} \frac{n_{e}}{n_{i}}=Z_{\mathrm{eff}}^{2} .
$$

Equation (6) shows that the electron and the ion conductivity are approximately of the same order, especially for singleionized gases, whenever the two fluids are equally distributed over the volume. In fact, for the last passage in Eq. (6), the relation $n_{e}=Z_{\mathrm{eff}} n_{i}$ has been considered. On the other hand, a Debye sheath arises in a plasma even when the electrons have a temperature comparable to that of the ions, since they are much lighter [20]. As already pointed out, for the plasma discharges of our interest we are going to consider the electrons and the ions at the same temperature since the thermalization through the electron-ion collisions occurs on a timescale much shorter than that of the discharge current. At the interface with the capillary surface, the electrons flow out of the plasma, charging the surface negative relative to the bulk plasma. Due to Debye shielding, the scale length of the transition region is proportional to the Debye length $\lambda_{D}=v_{\text {th }} / \omega_{p}$, where the electron thermal velocity is $v_{\text {th }}=$ $\left(k_{B} T / m_{e}\right)^{1 / 2}$. The electrostatic Debye sheath is a layer in the plasma with a greater density of positive ions, and hence an overall excess of positive charge, that balances an opposite negative charge on the surface of the material with which it is in contact. The thickness of such a layer is several Debye lengths thick, depending on the electron temperature and density [20]. The Debye sheath in the case of the plasma discharge capillary is placed at the interface between the bulk plasma and the capillary internal surface. Since the electrons are the main cause of the mass transfer, i.e., heat, toward the walls of the capillary, at the plasma-capillary interface $\kappa_{e} \gg$ $\kappa_{i}$. Moreover, since the Debye length is normally much shorter than the capillary radius $R_{\text {cap }}$, Eq. (4) can be straightforwardly reduced to

$$
H_{w} \sim \kappa_{e} T \frac{S_{\text {cap }}}{N_{D} \lambda_{D}} .
$$

In fact, the transition from the bulk temperature $T$ to the wall temperature $T_{0} \ll T$ is very sharp, therefore $\vec{\nabla} T \sim$ $\left(T-T_{0}\right) / N_{D} \lambda_{D} \sim T / N_{D} \lambda_{D}$. The number $N_{D}$ of Debye lengths that determines the variation scale of the heat flux to the walls is roughly given by the ratio $N_{D} \sim T_{c} / T_{0}$, since the number of plasma electrons lost into the walls is $\sim\left(T_{c} / T_{0}\right) n_{c} 2 \pi L_{\text {cap }} R_{\text {cap }} \lambda_{D}$ (with $n_{c}$ and $T_{c}$ the electron density at the center of the capillary), which before the onset of the sheet mechanism were distributed over a circular corona of width $\sim T_{c} / T_{0} \lambda_{D}$. An estimation of the temperature at the 
center of the capillary can be performed by using the analytic model presented in Sec. VI.

\section{B. Radiation rates}

Concerning the radiation losses, we first introduce the bremsstrahlung power radiated out of the plasma [21],

$$
P_{\mathrm{BR}}=\frac{\sqrt{k_{B} T} n_{e} n_{i} Z_{\mathrm{eff}}^{2} e^{6}}{3 \sqrt{2} \pi^{5 / 2} \varepsilon_{0}^{3} c^{3} m_{e}^{3 / 2} \hbar} V,
$$

and then the radiative recombination power $P_{\mathrm{RR}}$ emitted by the overall plasma volume [21],

$$
P_{\mathrm{RR}}=\left(\sum_{n=1}^{\infty} \frac{1}{n^{3}}\right) \frac{Z_{\mathrm{eff}}^{2} \mathrm{Ry}}{k_{B} T} \frac{2 \sqrt{k_{B} T} n_{e} n_{i} Z_{\mathrm{eff}}^{2} e^{6}}{3 \pi^{3} \varepsilon_{0}^{3} c^{3} m_{e}^{3 / 2} \hbar} V,
$$

where the sum of the infinite series gives approximately $\sim 1.2$. The unit energy Ry corresponds to $13.6 \mathrm{eV}$. By observing that the ratio $P_{\mathrm{RR}} / P_{\mathrm{BR}}$ is $\sim 2 Z_{\text {eff }}^{2} \mathrm{Ry} / k_{B} T$, it is possible to notice that for plasma temperatures of the order of a few $\mathrm{eV}$, the radiative recombination contribution to the losses is far greater than the bremsstrahlung one, and also that the radiation losses are more important for highly ionized gases.

\section{MULTI-IONIZATION, GAS MIXTURES, AND DEBYE SHIELDING}

The model developed in the present paper is based on general assumptions, and it can thus be applied to any atomic or molecular gas or even to mixtures. Starting from that, we introduce the Saha equation [18]

$$
\frac{n_{i} n_{e}}{n_{n}}=\left(\frac{k_{B} m_{e} T}{2 \pi \hbar^{2}}\right)^{3 / 2} \frac{2 g_{i}}{g_{i-1}} e^{-\frac{U_{i}}{k_{B} T}}
$$

where $g_{i}$ is the degeneracy factor of the $i$ th ionization level with ionization potential $U_{i}$. The Saha equation can only hold for plasmas in local thermodynamic equilibrium (LTE), which is valid mainly for plasmas where collisional processes are faster and more important than dissipative processes. For the case of interest here, as shown later in the paper, the time for electron-ion collisions and local thermalization is of the order of a few picoseconds, while the dissipation of energy through heat and radiation occurs over the plasma lifetime of the order of $\lesssim 1 \mu$ s. We define the total initial atom density as $n_{a}$, which is related to the electron $n_{e}$, ion $n_{i}$, and neutral densities as $n_{i}=Z_{\mathrm{eff}} n_{a}, n_{e}=n_{i}$, and $n_{n}=n_{a}-n_{e}$.

\section{A. Effective ionization degree}

The effective degree of ionization is

$$
Z_{\mathrm{eff}}=\frac{1}{2} \sum_{i}\left(-Z_{i}+\sqrt{4 Z_{i}+Z_{i}^{2}}\right),
$$

where

$$
Z_{i}=\frac{1}{n_{a}}\left(\frac{k_{B} m_{e} T}{2 \pi \hbar^{2}}\right)^{3 / 2} \frac{2 g_{i}}{g_{i-1}} e^{-\frac{U_{i}}{k_{B} T}} .
$$

It is worth noting that in this framework, the ion density $n_{i}$ is not the physical number density of ions within the gas, but rather the number of ionic states within the gas. This allows a simplified description of the ionization dynamics, justified by the fact that, for the purposes of this paper, plasma electron dynamics is much more relevant and interesting than plasma ion dynamics. Moreover, it is always possible to relate the density of ionic states to the plasma ion density for a particular ionization level by partitioning over the ionized levels through Eq. (11). The electron plasma density $n_{e}$ corresponds instead to the real density of plasma electrons within the capillary. This approach allows us to consider any kind of atomic or molecular gas, as far as the proper ionization potentials are taken into account.

\section{B. Case of gas mixtures}

The generalization to mixtures of gases is straightforward, since the total effective degree of ionization will be the sum of $Z_{\text {eff }}^{n}$, the effective degree of ionization of the $n$th gas, over all the gases composing the mixture

$$
Z_{\mathrm{eff}}^{\mathrm{tot}}=\sum_{n} Z_{\mathrm{eff}}^{n},
$$

and the total electron density will be

$$
n_{e}=\sum_{n} n_{a}^{n} Z_{\mathrm{eff}}^{n}
$$

where $n_{a}^{n}$ is the initial atom density of the $n$th gas of the mixture, depending on its initial pressure $P_{n}$ inside the capillary. The presence of more than one gas implies also the possible presence of more than one temperature, one relative to each gas. In this case, Eq. (2) becomes a system of equations, each of which referrs to one gas of the mixture, with its own evolution in time. The equations of the system will be coupled by interaction terms of the kind $v_{i j} \Delta T_{i j}$ (with $v_{i j}$ the collision rate between two molecular species $i$ and $j$, and $\Delta T_{i j}$ the temperature difference between them), which take into account the gas-gas thermal exchange through collisions. Other coupling terms would be radiation loss terms relating to the recombination of electrons over different species of ions as well as to bremsstrahlung losses due to collisions between electrons and ions of different species. Diffusion dynamics could be neglected in the sense that the model proposed considers an average of all quantities of interest over the plasma volume, instead of local magnetohydrodynamic phenomena.

\section{Debye shielding in plasmas}

Concerning the Debye shielding in plasmas, i.e., the effect of shielding of local electric fields due to the plasma electrons surrounding plasma ions, we must remember that this can bring about a reduction of the ionization potential. The Coulomb potential of a plasma ion is actually modified by the Debye term of the form $\exp \left(-r / \lambda_{D}\right)$. When the Debye length is comparable to the atomic radius $r_{i}$ relative to the $i$ th energy level, the attractive potential that keeps the atomic electrons bound is reduced. Therefore, for $r_{i} \ll \lambda_{D}$, which is the condition in which we are mostly interested, the ionization potential $U_{i}$ must be replaced, $U_{i} \rightarrow U_{i}\left(1-r_{i} / 2 \lambda_{D}\right)$. For plasmas with a temperature of a few $\mathrm{eV}$, this correction is of the order of a few percent when the electron plasma density 
is about $\sim 10^{17} \mathrm{~cm}^{-3}$, and of the order of $10 \%$ for electron plasma densities around $10^{19} \mathrm{~cm}^{-3}$.

\section{PLASMA RESISTIVITY}

A nonionized gas is not conductive, therefore its resistivity tends to "infinite." For a gas in its neutral conditions, collisions happen only between atoms and electrons. In gases, in fact, there is always a very low percentage of free electrons, due to natural reasons such as heat exchange with the environment and environmental radiation. The resistivity of a gas evolving into a plasma can be written as $[15,16]$

$$
\rho_{\mathrm{tot}}=\rho_{e i}+\rho_{a e}=\frac{m_{e}}{n_{e} e^{2}}\left(v_{e i}+v_{a e}\right),
$$

where collisions between electrons and ions occur at a rate $v_{e i}$, and $\rho_{a e}$ is the resistivity due to the collisions between atoms and electrons (occurring at a rate $v_{a e}$ ). The electron-atom collision rate is defined as $v_{a e}=v_{\text {th }} \sigma_{a e} n_{a}$, where $\sigma_{a e}$ is the cross section for electron-atom collisions:

$$
\sigma_{a e}=\pi r_{a}^{2},
$$

where $r_{a}$ is the atomic radius. Considering a Maxwellian distribution for the electron velocities, and the fact that $n_{a}=$ $P_{0} / k_{B} T$, we have

$$
v_{a e}=\frac{\pi r_{a}^{2} P_{0}}{\sqrt{m_{e} k_{B} T}} .
$$

The electron-ion collision rate instead is calculated as [21]

$$
v_{e i}=\frac{n_{i} Z_{\mathrm{eff}}^{2} e^{4}}{2 \pi \varepsilon_{0}^{2} m_{e}^{2} v_{\mathrm{th}}^{3}} \ln \Lambda,
$$

where the Coulomb logarithm is

$$
\ln \Lambda=\ln \left(\frac{b_{\max }}{b_{\min }}\right)=\ln \left(\frac{\lambda_{D}}{b_{\min }}\right) .
$$

The Coulomb logarithm takes into account the minimum and maximum impact parameter ( $b_{\min }$ and $b_{\max }$, respectively) between plasma electrons and plasma ions. In fact, the Debye length is the maximum impact parameter, since the Coulomb field rapidly decays further than that distance from the ion, while the minimum impact parameter is the maximum between the turning point of the electromagnetic collision and a fraction of the de Broglie wavelength [22], i.e.,

$$
b_{\min }=\max \left\{\frac{Z_{\mathrm{eff}} e^{2}}{2 \pi \varepsilon_{0} m_{e} v_{\mathrm{th}}^{2}}, \frac{\hbar e^{-\gamma}}{m_{e} v_{\mathrm{th}}}\right\},
$$

where $\gamma$ is the Eulero-Mascheroni constant. Plasma resistivity due to collisions is therefore given by

$$
\rho_{\mathrm{tot}}=\frac{m_{e}}{n_{e} e^{2}} \frac{n_{i} Z_{\mathrm{eff}}^{2} e^{4}}{2 \pi \varepsilon_{0}^{2} m_{e}^{2} v_{\mathrm{th}}^{3}} \ln \Lambda+\frac{m_{e}}{n_{e} e^{2}} \frac{n_{n}}{n_{a}} \frac{\pi r_{a}^{2} P_{0}}{\sqrt{m_{e} k_{B} T}} .
$$

Finally, the plasma resistance, assuming for the sake of simplicity a uniform plasma inside the capillary, is

$$
R_{p}=\left(\rho_{e i}+\rho_{a e}\right) \frac{L_{\text {cap }}}{S_{\text {cap }}} .
$$

Even in the presence of nonperfectly uniform plasmas, our assumption can represent the bulk of plasma up to a geometrical factor, on the right-hand side of Eq. (22), of the order

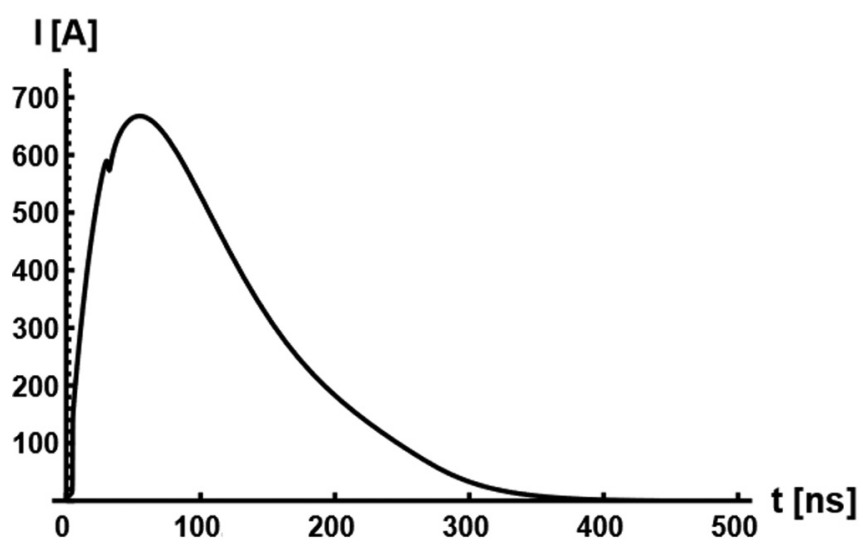

FIG. 1. Plot of the discharge current flowing in the capillary.

of unity. Therefore, we consider Eq. (22) as an acceptable approximation within the scope of the paper. The presence of both electron-atom and electron-ion collision terms in the microscopic definition of the plasma resistivity ensures the gas breakdown and the flow of a current pulse through the formed plasma. Indeed, the gas resistivity, infinite at the beginning, starts to decrease tending to zero when the temperature dissipated into the plasma resistor becomes responsible for atom ionization and plasma formation. Then the dominant resistivity term is the plasma resistivity, which is very low since the high conductivity is due to the free plasma electrons. Finally, according to the circuit parameter $(R, L, C)$, it is possible to individuate a strongly damped regime of oscillation (namely the discharge regime, which is the most interesting for this paper) and also other regimes (oscillatory and slightly damped oscillatory), where the potential drop is not totally dissipated into the plasma in the form of heating, but it oscillates between electrostatic and magnetostatic energy back and forth from the capacitance to the inductance.

\section{SIMULATION RESULTS}

Two sets of simulations are going to be shown in the following. The first ones refer to a discharge onset within a hydrogen plasma. Hydrogen is the simplest gas existing in nature, and the aim is to show the reliability of the results provided by our simplified code. In particular, we focus on the loss mechanisms, individuating which ones are dominant and which are not. The second set of simulations show the comparison among different gases, coupled with the same discharge circuit. This is another important example that can shed light on the physics described by the model.

\section{A. Hydrogen plasma}

The first simulations have been performed with the following parameters for the discharge: $20 \mathrm{kV}$ discharge potential, $20 \Omega$ parasitic resistor, $600 \mathrm{nH}$ parasitic inductance, and $5 \mathrm{nF}$ circuit capacitance. For the gas, as was already pointed out, molecular hydrogen has been considered, with an initial pressure of 10 mbar. The capillary internal radius is $500 \mu \mathrm{m}$ and the capillary length is $5 \mathrm{~cm}$. Figure 1 shows the simulation of a discharge current flowing inside the capillary embedding 


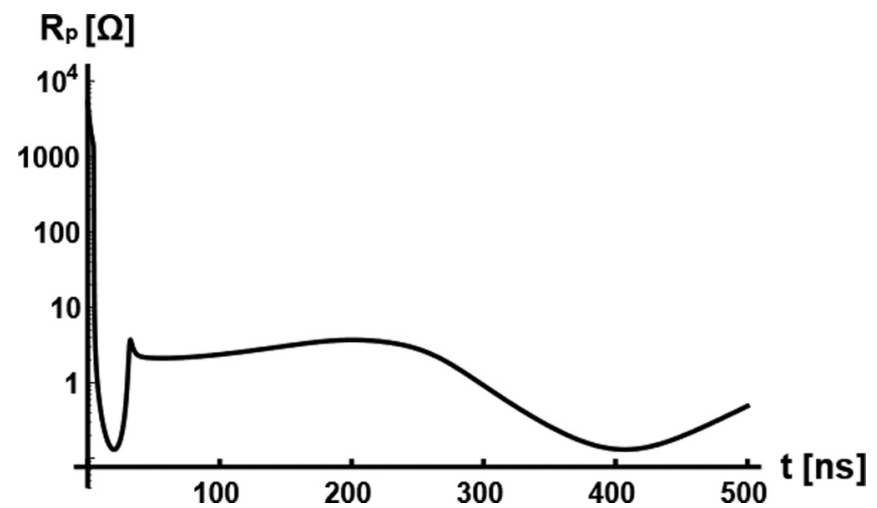

FIG. 2. Plot of the plasma resistance evolution.

the hydrogen gas. The peak current for our parameters is around $660 \mathrm{~A}$ and the duration is between 100 and $200 \mathrm{~ns}$. Figure 2 shows the plasma resistance drop over time. The initial condition of infinite resistance is due to the absence of plasma inside the capillary, with the gas still being a perfect insulator. The inversion of trend around $20 \mathrm{~ns}$ after an initial breakdown is due to a noncomplete ionization of the gas. The number of neutrals starts to increase again due to recombination, causing also a drop in the discharge current (Fig. 1) and a bump in the temperature evolution versus time (Fig. 3). After the level of ionization has reached its maximum and the gas is almost completely ionized, the resistance shows a plateau (also visible in Fig. 5 showing the electron plasma density). The plasma temperature shows a maximum temperature $\sim 3.5 \mathrm{eV}$, sufficient for a complete ionization of the hydrogen molecules, with ionization potential $\sim 15.4 \mathrm{eV}$ as shown in Table I. Figure 4 shows the rate of collisions between plasma electrons and plasma ions, much faster than the discharge lifetime. At the beginning, the collision rate is zero since there is no plasma in the capillary, and then it rapidly reaches the value of $\sim 1 \mathrm{THz}$, meaning that our assumption of a unique plasma temperature is reasonable, since within the timescale of the discharge current the number of collisions thermalizing the two fluids is high enough. Figure 5 shows the evolution of the average electron plasma density. The initial density of gas particles is $P_{0} / k_{B} T_{0} \sim 2.4 \times 10^{17} \mathrm{~cm}^{-3}$, and the electron plasma density is two times this value, consistent with the fact that we are considering molecular hydrogen. Finally, the different contributions on the right side of Eq. (2) are shown in Fig. 6. The heat losses into the walls are the most important, and an equilibrium is reached with the Ohmic plasma heating due to the current flow inside the plasma over roughly $150 \mathrm{~ns}$. The radiation losses, particularly those related to the radiative recombination, also participate in the thermal

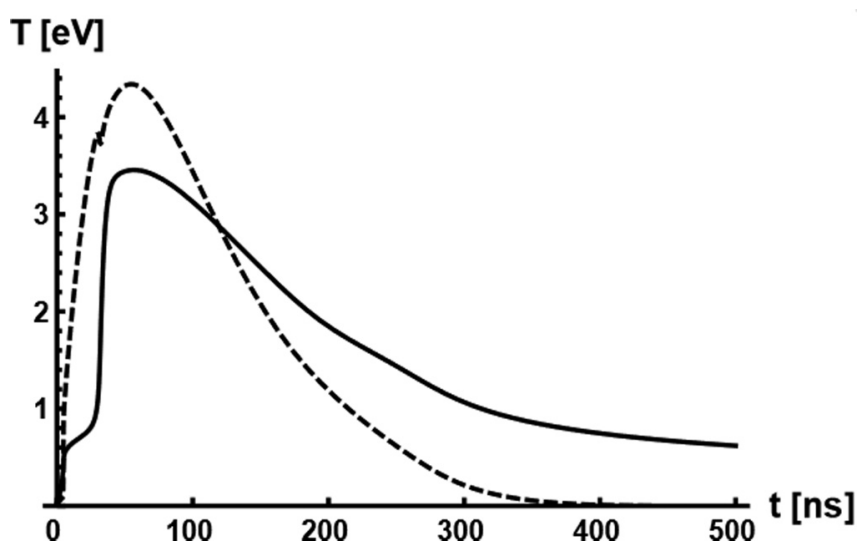

FIG. 3. Plot of the average (over the capillary volume) plasma temperature evolution. Superimposed as a dashed curve is the discharge current's profile of Fig. 1 (arbitrary units).

equilibrium even if it is as a higher-order correction. This aspect will be addressed in detail in Sec. VI.

\section{B. Comparison among different gases}

The second set of simulations shows the differences in the plasma parameters among different gases, given the same input discharge parameters, which, in particular, were chosen to be the same as those used for the hydrogen plasma simulations. Only the input gas pressure has been set two times higher, i.e., 20 mbar, for the atomic gases such as helium and argon. In fact, the studied gases were molecular hydrogen, molecular nitrogen, helium and argon. Figure 7 shows the plasma temperature evolution among the four gases under analysis. There are two important observations: (i) The plasma temperatures are all comparable to each other, consistent with the fact that the input energy from the discharge circuit is the same for all the gases. (ii) The only difference is that the onset of the plasma heating is delayed in time moving from the lighter to the heaviest gas. This effect is clearly related to the ion species under consideration, and in particular to the atomic radii via the electron-atom collision rate. Figure 8 shows a comparison of the discharge current among the different gases. Again, the peak values of the discharge currents are all comparable to each other, but the onset of the discharge is delayed in time going from hydrogen to argon. This is explained using Eq. (17), which shows that the elastic electron-atom collisions are greater when the atomic radius is higher. Thus, it takes longer for the plasma resistance to decrease, thus causing the onset of the discharge flow. Finally, the electron plasma density evolution is reported in Fig. 9. The actual values of the electron plasma density mirror the

TABLE I. Gas considered in this paper with the corresponding first five ionization potentials (truncated to the decimal digit).

\begin{tabular}{lcccc}
\hline \hline Gas & $\begin{array}{c}\text { First Ion. Pot. } \\
(\mathrm{eV})\end{array}$ & $\begin{array}{c}\text { Second Ion. Pot. } \\
(\mathrm{eV})\end{array}$ & $\begin{array}{c}\text { Third Ion. Pot. } \\
(\mathrm{eV})\end{array}$ & $\begin{array}{c}\text { Fourth Ion. Pot. } \\
(\mathrm{eV})\end{array}$ \\
Hydrogen & 15.4 & & & $\begin{array}{c}\text { Fifth Ion. Pot. } \\
(\mathrm{eV})\end{array}$ \\
Helium & 24.6 & 54.4 & 47.5 & 77.5 \\
Nitrogen & 15.6 & 29.6 & 40.7 & 59.8 \\
Argon & 15.8 & 27.6 & 75.0 \\
\hline \hline
\end{tabular}




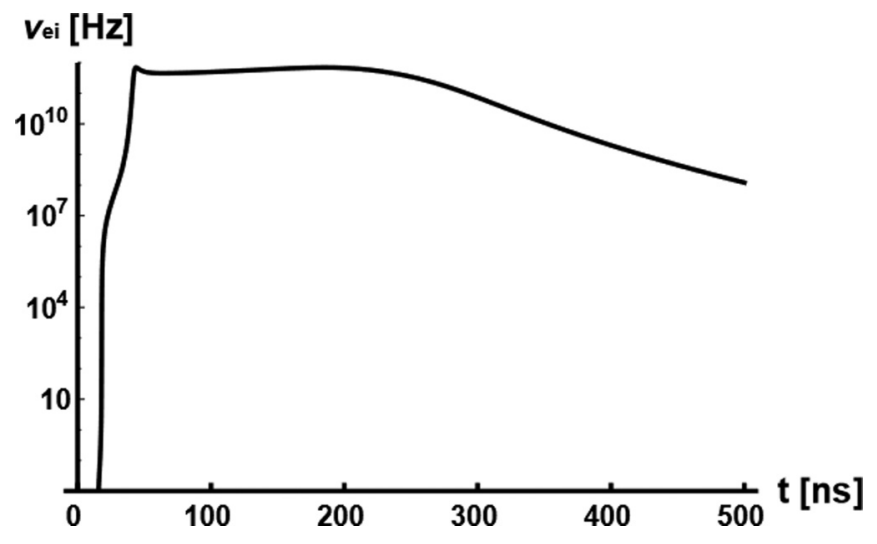

FIG. 4. Plot of the electron-ion collision rate evolution.

behavior of the degrees of ionization for each considered gas, as they should.

\section{RADIAL MODEL AT THE EQUILIBRIUM WITH RADIATION LOSSES}

In the present section, we aim to construct an analytic model that is more complete than the one presented in Ref. [13]. Indeed, as already shown in the previous section, there is a condition of thermal equilibrium inside the capillary between the Ohmic heating of the plasma and the other sources of loss. Mainly, the Ohmic heating is balanced by the heat loss into the capillary walls, but it is not always possible to neglect the energy that flows away from the capillary (cooling down the plasma) through radiative means. Therefore, with the goal of taking into account radiation losses, and recalling that by virtue of the perfect gas equation of state the electron plasma density scales as the inverse of the temperature, we get a local evolution equation of the temperature along the transverse plane of the capillary,

$$
\frac{1}{\xi} \frac{\partial}{\partial \xi}\left(\xi \frac{\partial u}{\partial \xi}\right)=-u^{3 / 7}+\varrho u^{-1 / 7}
$$

where $\xi=r / R_{\text {cap }}$ and the plasma temperature is $T=A u^{2 / 7}$, with $A=\left(7 R_{\text {cap }}^{2} \sigma_{0} E_{0}^{2} / 2 \kappa_{0}\right)^{1 / 2}$. The definition of the constant $A$ is the same as in Ref. [13], as well as the definition of all the

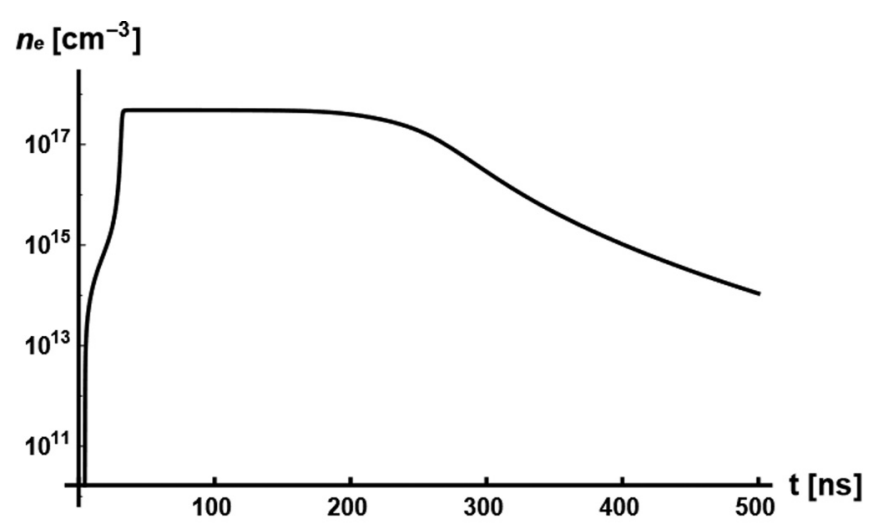

FIG. 5. Plot of the evolution of the average (over the capillary volume) electron plasma density.

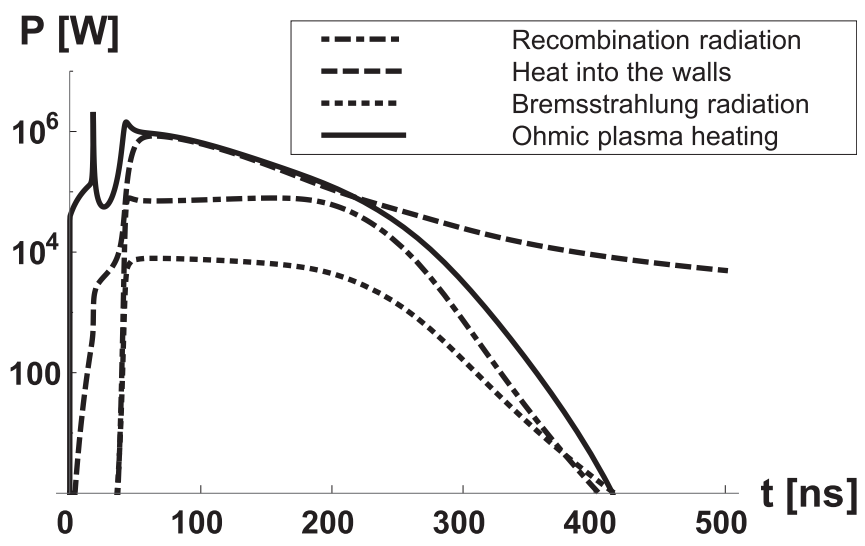

FIG. 6. Plot of the different contributions on the right side of Eq. (2).

constants appearing in its formula. In summary, $\sigma_{0}$ and $\kappa_{0}$ are the parts of the electric and thermal conductivity excluding the temperature, namely $\sigma=\sigma_{0} T^{3 / 2}$ and $\kappa=\kappa_{0} T^{5 / 2}$, while $E_{0}$ is the electric field along the capillary due to the current flow. Here we have introduced a new parameter, the $\varrho$ constant, which takes into account the correction to the temperature profile at the equilibrium due to radiation losses (radiative recombination only),

$$
\varrho=\left(\sum_{n=1}^{\infty} \frac{1}{n^{3}}\right) \frac{4}{21 \pi^{3}} \frac{\kappa_{0} \mathrm{Ry} P_{0}^{2} e^{6}}{\sigma_{0}^{2} E_{0}^{4} R_{\mathrm{cap}}^{2} k_{B}^{5 / 2} T_{0}^{2} \varepsilon_{0}^{3} c^{3} m_{e}^{3 / 2} \hbar} .
$$

Equation (24) strictly refers, for the sake of simplicity, to fully single-ionized plasmas, otherwise, based on Eq. (9), a factor $Z_{\mathrm{eff}}^{6}$ should be estimated and taken into account as well. Furthermore, it is convenient to consider a weighted average of the electric field $E_{0}$, following the definition in [13]. We have estimated the electron plasma density as $\sim P_{0} / k_{B} T_{0}$. It is important to notice that Eq. (23) holds only for $-u^{3 / 7}+$ $\varrho u^{-1 / 7} \leqslant 0$. In fact, the condition $-u^{3 / 7}+\varrho u^{-1 / 7}>0$ would violate thermodynamics. The maximum value of $\varrho$ for which our model holds is $\varrho_{\max } \sim 7.5 \times 10^{-2}$. Thus, the inequality $\varrho<\varrho_{\max }$ set a condition for the maximum pressure that can be used in a plasma discharge capillary. The condition $\varrho=\varrho_{\max }$ corresponds to a pressure such that the Ohmic heating is

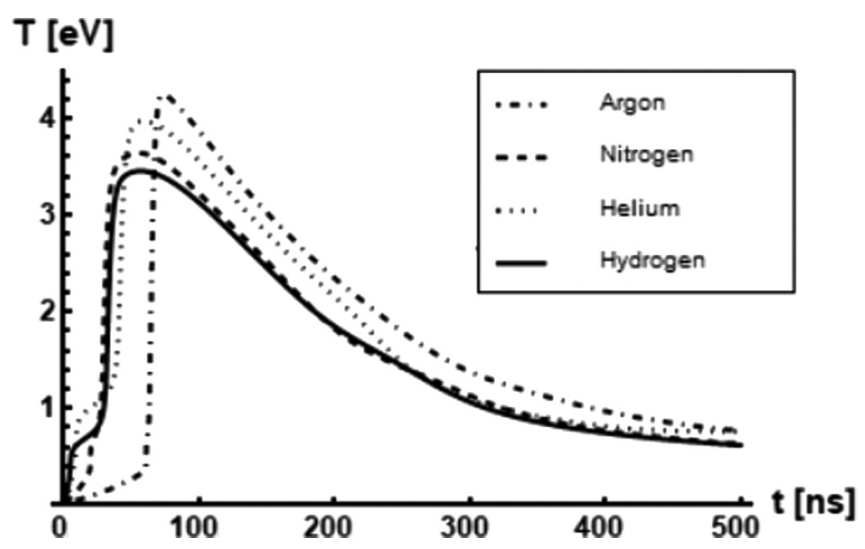

FIG. 7. Comparison of the plasma temperature among different gases, given the same input discharge parameters. 


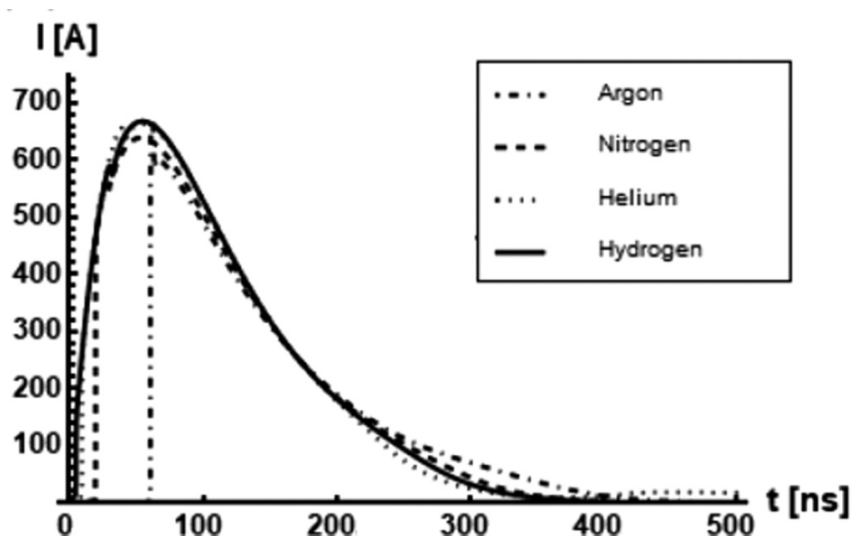

FIG. 8. Comparison of the discharge current among different gases, given the same input discharge parameters.

totally compensated by radiative losses and no increase of temperature occurs, i.e., no plasma heating or production. The condition $\varrho>\varrho_{\max }$ would correspond to the nonphysical situation when the radiative losses occur even without plasma formation and heating, which is contradictory. The temperature at the center of the capillary is $\varrho$-dependent: $T(0) \equiv T_{\varrho}(0)$. It is then possible to derive an expression for the temperature at the center of the capillary with radiative corrections

$$
T_{\varrho}(0) \sim A u(0)^{2 / 7} \times f(\varrho),
$$

where the calculated function $f(\varrho)$ is reported in Fig. 10.

\section{A. Numerical examples}

The modification to the function $u(\xi)$ as well as the difference in plasma temperature for the cases $\varrho=0$ and $\varrho=3.5 \times$ $10^{-2}$ are shown in Figs. 11 and 12, respectively. The value $\varrho=3.5 \times 10^{-2}$ refers to the first series of simulations shown in Sec. V A. The effect on plasma temperature and, indirectly, on all the other temperature-dependent quantities, such as the magnetic field associated with the discharge current (Fig. 13) and the electron plasma density (Fig. 14), is quite important even if the parameter $\varrho$ can look relatively small. The average value of plasma temperature over the transverse plane of the capillary with radiation correction is then $2.75 \mathrm{eV}$, in agree-

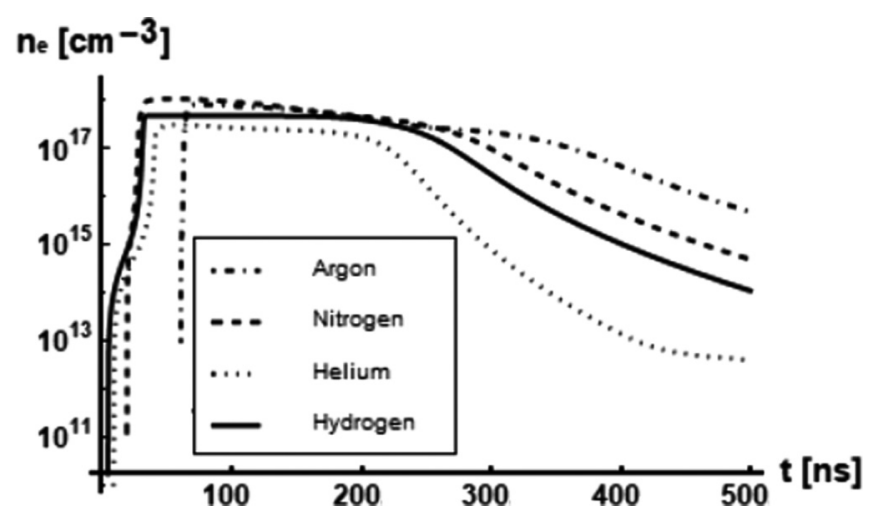

FIG. 9. Comparison of the electron plasma density among different gases, given the same input discharge parameters. $f(e)$

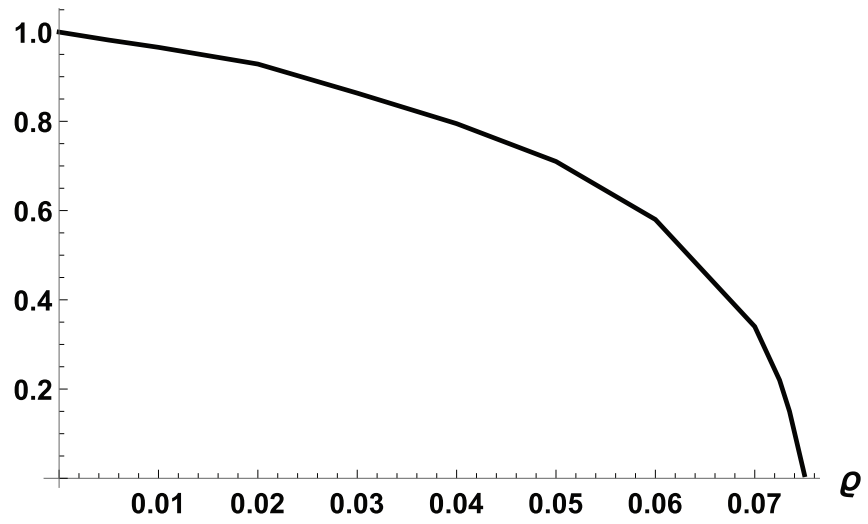

FIG. 10. Function $f(\varrho)$, expressing the dependence of $T(0)$ upon the $\varrho$ parameter.

ment with the average value of temperature in Fig. 3, over the time interval 50-200 ns in Fig. 6, i.e., $2.8 \mathrm{eV}$, which refers to the overlapping between the "Heat into the walls" curve with the "Ohmic plasma heating" power, i.e., to the thermal equilibrium. The average value of the plasma temperature over the transverse plane of the capillary without radiation correction is instead $3.3 \mathrm{eV}$, overestimating the correct temperature by about $20 \%$. Non-negligible effects of the same order can be observed on the radial slope of the magnetic field, which is useful for plasma lensing experiments, as well as on the radial profile of the electron plasma density, which is crucial for laser guiding in plasma channels.

\section{B. Laser guiding in plasma channels}

A laser beam can be efficiently guided inside a plasma channel when its beam radius is matched to the radial profile of the electron plasma density in the channel. According to the model shown in this paper, including thermal and radiation effects, and according to formulas reported in Ref. [13], the laser beam matched to the plasma channel must have the

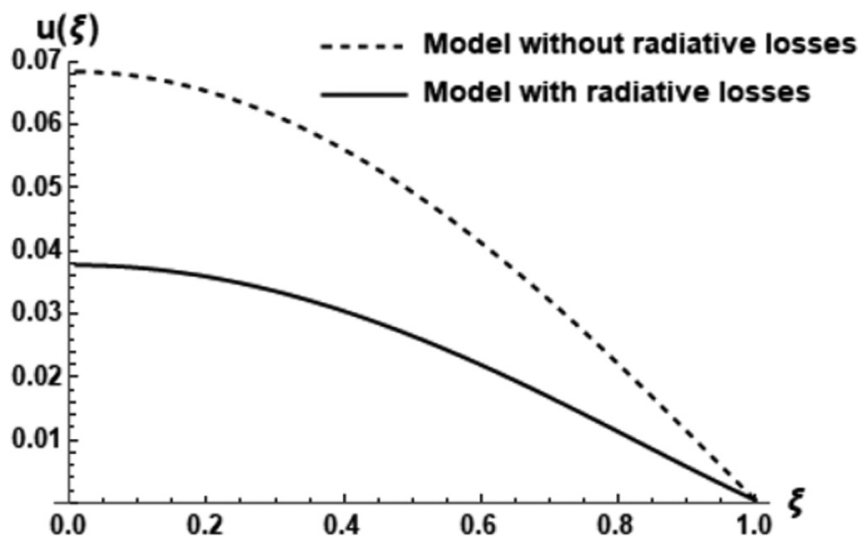

FIG. 11. Comparison between the solution $u(\xi)$ without radiative corrections (dashed line) and with radiative corrections (solid line) for the case $\varrho=3.5 \times 10^{-2}$. 


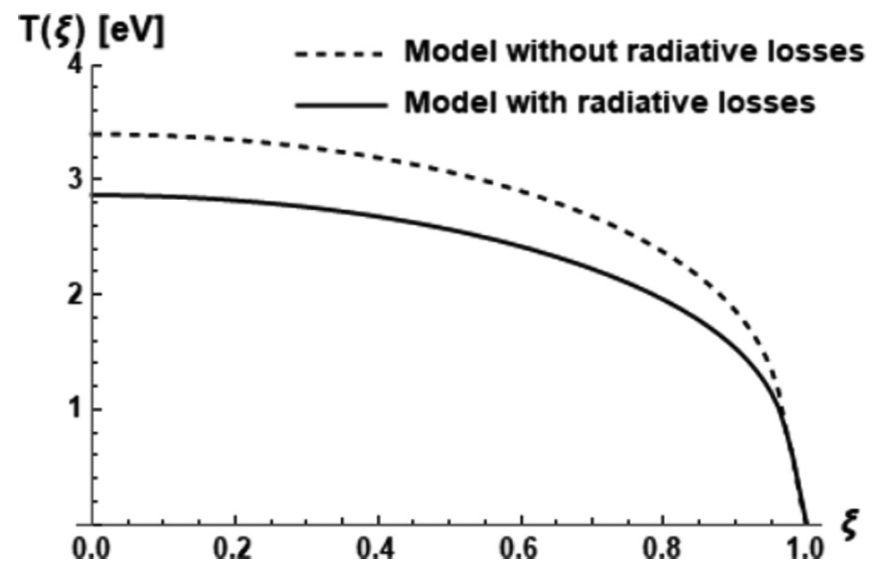

FIG. 12. Comparison between the plasma temperature's transverse profile without radiative corrections (dashed line) and with radiative corrections (solid line) for the case $\varrho=3.5 \times 10^{-2}$.

following spot size:

$$
W_{M}(\varrho)(\mu \mathrm{m})=1.48 \times 10^{5} \frac{\left[f(\varrho) R_{\text {cap }}(\mu \mathrm{m})\right]^{1 / 2}}{\left[Z_{\text {eff }} n_{a}\left(\mathrm{~cm}^{-3}\right)\right]^{1 / 4}},
$$

where the dependence upon the $\varrho$ parameter can be clearly seen. Furthermore, the effective ionization degree can be calculated using Eqs. (11) and (12) together with Eq. (25).

\section{A SIMPLE MODEL FOR STARK-BROADENING AND PLASMA DIAGNOSTICS IN THE VISIBLE RANGE}

Previously we have only considered the two main contributions (RR,BR) to the radiation emitted by a plasma discharge, since the aim was to study the energy exchange between the plasma and the environment. Nevertheless, plasma emission consists also of transition lines, even if it is with a smaller yield of photons. In particular, for hydrogen, the Balmer series is extremely important. In the case of atomic gases, the emission of atomic lines is due to the deexcitation of atomic levels higher than the ground state. The Balmer series of hydrogen concerns the quantum falls from any atomic state with principal number $>2$ down to the state $n=2$.

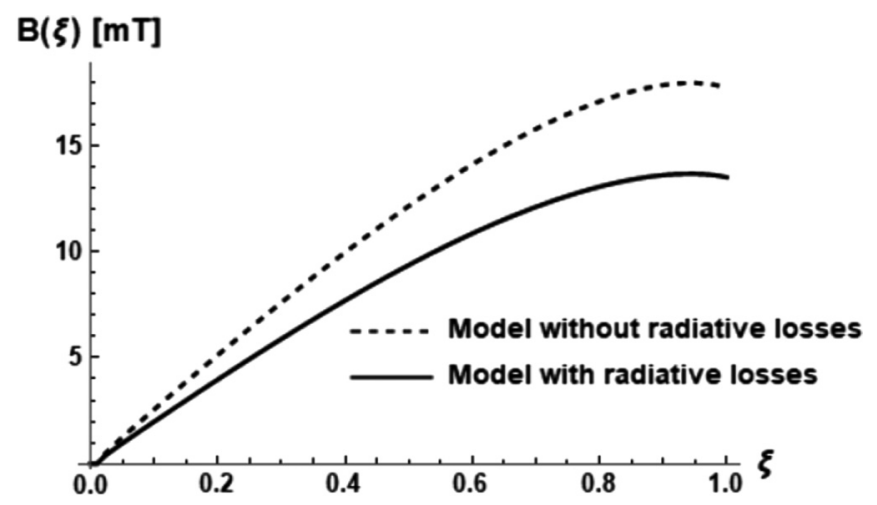

FIG. 13. Comparison between the magnetic field's (associated to the discharge current) transverse profile without radiative corrections (dashed line) and with radiative corrections (solid line) for the case $\varrho=3.5 \times 10^{-2}$.

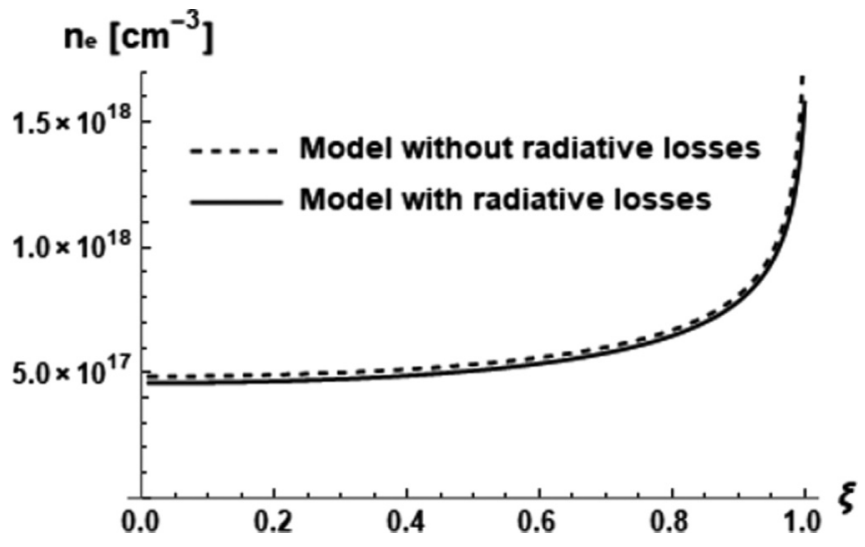

FIG. 14. Comparison between the electron plasma density's transverse profile without radiative corrections (dashed line) and with radiative corrections (solid line) for the case $\varrho=3.5 \times 10^{-2}$.

The dominant contribution to the width of the Balmer lines, for the plasma electron densities studied in this work, is given by the mechanism of Stark-broadening [23], i.e., the effect of broadening of emission lines due to the presence of an external and static electric field. The microscopic field, acting in the vacuum interspace among the hydrogen plasma electrons, must vary over a distance that is half of the density scale length $\sim n_{e}^{-1 / 3}$, thus

$$
\vec{\nabla} \cdot \vec{E} \sim-2 n_{e}^{1 / 3} E=-\frac{e n_{e}}{\varepsilon_{0}} .
$$

In fact, the local electric field is maximum in the vicinity of the plasma electrons, and then approaches zero (for symmetry reasons) at half of the electrons' interdistance $n_{e}^{1 / 3}$. The excited atoms inside the plasma do experience this electric field when in proximity to the plasma electrons, and their atomic dipole moments $p_{\text {at }}$ can couple to it through the electric dipole coupling, whose absolute value is $H_{\mathrm{dip}} \sim p_{\mathrm{at}} E$. The atomic dipoles for the Balmer- $\alpha$ line can be estimated as

$$
p_{\text {at }}=p_{\alpha} \sim-e \overline{\left|\left\langle 2, l, m_{l}|\vec{r}| 3, l, m_{l}\right\rangle\right|} \sim-e a_{B},
$$

where $a_{B}$ is the Bohr radius, with the overline indicating both a summation over the orbital and magnetic quantum numbers $l, m_{l}$ and a quadratic average over the nonzero values of the electric dipole. Expression (28) refers to all the possible transitions between the levels with principal quantum number 3 to the levels labeled by the principal quantum number 2. Hydrogen wave functions, solutions of the Schrödinger equation for a Coulomb potential, have been used for calculating the right-hand side of Eq. (28), despite the triviality of the final result. The Balmer- $\alpha$ transition energy $\hbar \omega_{\alpha}$ is Stark-broadened in plasmas as $\hbar \omega_{\alpha} \pm\left|p_{\alpha} E\right|$, depending on whether the dipoles are aligned or antialigned to the electric field. In terms of the wavelength $\lambda_{\alpha}=2 \pi c / \omega_{\alpha}$, the Balmer- $\alpha$ Stark-broadening can be found as

$$
\Delta \lambda_{\alpha}\left(n_{e}\right)=\frac{p_{\alpha} e \lambda_{\alpha}^{2}}{2 \pi \varepsilon_{0} \hbar c} n_{e}^{2 / 3}
$$

This expression is consistent within a few percent of relative error with previous works on the topic [23-25], where $\Delta \lambda_{\alpha}$ 
is understood here to be two times the rms width of the Balmer- $\alpha$ line. In general, the line-broadening can also be due to a Doppler mechanism, from which the width $\Delta \lambda_{\alpha}\left(n_{e}\right) \rightarrow$ $\Delta \lambda_{\alpha}\left(n_{e}, T\right)$ also depends on the electron plasma temperature. Nevertheless, since this dependence is weak for the density and temperature ranges explored in this paper, the correctness of Eq. (29) is guaranteed to the percent level, and the Doppler effect is negligible with respect to the Stark effect. The Balmer- $\alpha$ wavelength is $\lambda_{\alpha} \sim 656 \mathrm{~nm}$ : the electron plasma density in terms of the Stark-broadening of $\lambda_{\alpha}$ is obtained by solving Eq. (29) in terms of $n_{e}$.

\section{A. Stark-broadening of spontaneous electric dipoles}

By exploiting a hydrogenlike model, it is possible to generalize the diagnostic method to other gases with a spontaneous electric dipole like some of the ones explored above. In fact, considering the transition from principal quantum levels $n=$ 3 down to $n=2$ states and making the substitution $a_{B} \rightarrow$ $a_{B} / Z_{s}$, it is possible to use Eq. (29) to retrieve the electron plasma density for gases different from hydrogen. Here we have introduced $Z_{S}$, i.e., a screened atomic number satisfying the equation

$$
\frac{1}{2} \alpha Z_{s}^{2} m_{e} c^{2}\left(\frac{1}{2^{2}}-\frac{1}{2^{3}}\right)=\hbar \omega_{3 \rightarrow 2}
$$

where $\hbar \omega_{3 \rightarrow 2}$ is the energy corresponding to the transition from the level with principal quantum number 3 to the level with principal quantum number 2. For example, considering the helium plasma emission spectrum, the wavelength corresponding to the $\hbar \omega_{3 \rightarrow 2}$ photon is placed at $\sim 501 \mathrm{~nm}$. For this transition, the screened atomic number is found as $Z_{s} \sim 1.15$, according to Eq. (30).

\section{B. Stark-broadening of induced electric dipoles}

Generalizing the method to molecular gases such as $\mathrm{N}_{2}$ with no spontaneous electric dipole is not trivial since the spectrum is dominated by molecular transitions and the theory developed above for atomic transitions (in particular, we have considered the $3 \rightarrow 2$ transition) cannot hold. Focusing on molecular nitrogen, the average electric dipole, induced via electric polarizability, can be expressed as

$$
p_{\mathrm{N}_{2}}=\alpha_{\mathrm{N}_{2}} \frac{e n_{e}^{2 / 3}}{2 \varepsilon_{0}},
$$

where the experimentally determined polarizability is $\alpha_{\mathrm{N}_{2}} \sim$ $1.9 \times 10^{-40} \mathrm{C} \mathrm{m}^{2} \mathrm{~V}^{-1}$. In this case, we can still set an equation that can help to retrieve the electron plasma density from the measurement of the Stark-broadened lines belonging to some particular $\mathrm{N}_{2}$ multiplet $\lambda$,

$$
\Delta \lambda_{\mathrm{N}_{2}}\left(n_{e}\right)=\frac{\alpha_{\mathrm{N}_{2}} e^{2} \lambda^{2}}{4 \pi \varepsilon_{0}^{2} \hbar c} n_{e}^{4 / 3} .
$$

For molecular transitions, the scaling of the Stark width is $n_{e}^{4 / 3}$ instead of $n_{e}^{2 / 3}$ for atomic transitions.
Signal [arb. units]

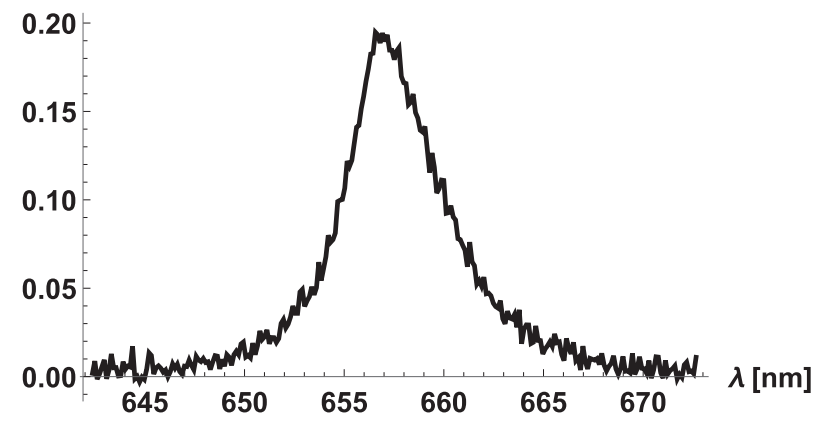

FIG. 15. Measurement of the Stark-broadened hydrogen Balmer$\alpha$ line.

\section{Experimental results and simulations}

An experimental example of the Stark-broadened hydrogen Balmer- $\alpha$ line is reported in Fig. 15. The corresponding electron plasma density obtained by use of Eq. (29) is $n_{e} \sim$ $2.5 \times 10^{18} \mathrm{~cm}^{-3}$. The analogous measurement for a helium plasma is reported in Fig. 16, with a corresponding electron density retrieved, $n_{e} \sim 1.4 \times 10^{18} \mathrm{~cm}^{-3}$. Figure 17 shows experimental results on the Stark-broadened molecular nitrogen emission lines corresponding to the multiplet $3 s^{4} P-4 p^{4} S^{0}$ (nominally $\lambda=413.764,414.343$, and $415.148 \mathrm{~nm}$ ). Due to the poor resolution of the spectrometer $\sim 1 \AA$, it has been possible only to state that the rms width of the line placed at $413.764 \mathrm{~nm}$ was $\Delta \lambda_{\mathrm{N}_{2}}<0.5 \AA$. With this information, it has been possible to set an experimental threshold to the measured electron plasma density $n_{e}<9 \times 10^{18} \mathrm{~cm}^{-3}$. Spectroscopic data shown in this section do not have time resolution, thus they have to be considered as referring to time averages of the electron plasma density. The collection of light was occurring over the whole plasma lifetime and within a solid angle covering the full region of light emission from one side of the capillary, including the region of the expanding plasma and the region of the gas inlets (see Sec. VIID). Due to the scaling for line emission as $\propto n_{e}^{2}$ [21], light emission occurs mainly during the time (and from the volume) of maximal density. Therefore, the electron plasma density measured using this method is automatically the electron plasma density weighted on the power of line emission. Figure 19 shows simulations of electron plasma density, based on the model described in

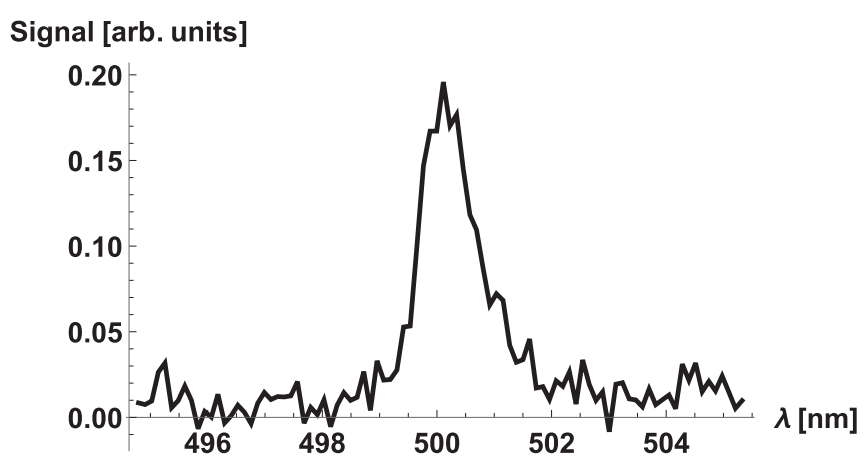

FIG. 16. Measurement of a Stark-broadened helium emission line corresponding to the atomic transition $3{ }^{1} \mathrm{P} \rightarrow 2{ }^{1} S$. 


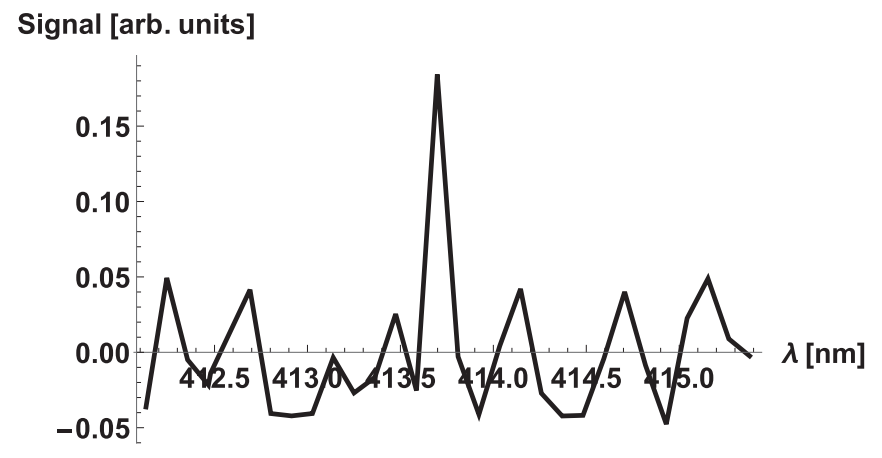

FIG. 17. Measurement of Stark-broadened molecular nitrogen emission lines corresponding to the multiplet $3 s^{4} P-4 p^{4} S^{0}$ : measurement limited by the spectrometer's resolution.

Sec. II, referring to the experimental data just presented and obtained at the FLAME laser facility [17]. The discharge parameters in this case were as follows: $20 \mathrm{kV}$ discharge potential, $40 \Omega$ parasitic resistor, $600 \mathrm{nH}$ parasitic inductance, and $10.8 \mathrm{nF}$ circuit capacitance. A schematic of the real discharge circuit used for the experiment is provided by Fig. 18, equivalent to an RLC-series circuit with parameters summarized above. The schematic of the experimental setup was such that the plasma discharge capillary was placed on a hexapod; the spatial distribution of the discharge was monitored by a CCD camera, and the spectral content of the plasma light, used for measurements of electron plasma density, was recorded by means of a fiber spectrometer. For the gases, a pressure of 52.5 mbar was set. The capillary internal radius was $250 \mu \mathrm{m}$ and the capillary length $3 \mathrm{~cm}$. When considering the average density over the whole plasma lifetime, all the simulated electron densities are in good agreement with the measured ones, based on the simple analytic model correlating the Stark-broadened width of the gas emission lines with the electron plasma density, both for atomic and molecular transitions. The simulated average density $\bar{n}_{e}$ was calculated as the weighted average on the instantaneous amount of light:

$$
\bar{n}_{e}=\frac{\int_{0}^{\infty} n_{e}^{3} d t}{\int_{0}^{\infty} n_{e}^{2} d t} .
$$

The weighted average density obtained from hydrogen simulations (Fig. 19) was $\bar{n}_{e}=2.3 \times 10^{18} \mathrm{~cm}^{-3}$, the one obtained

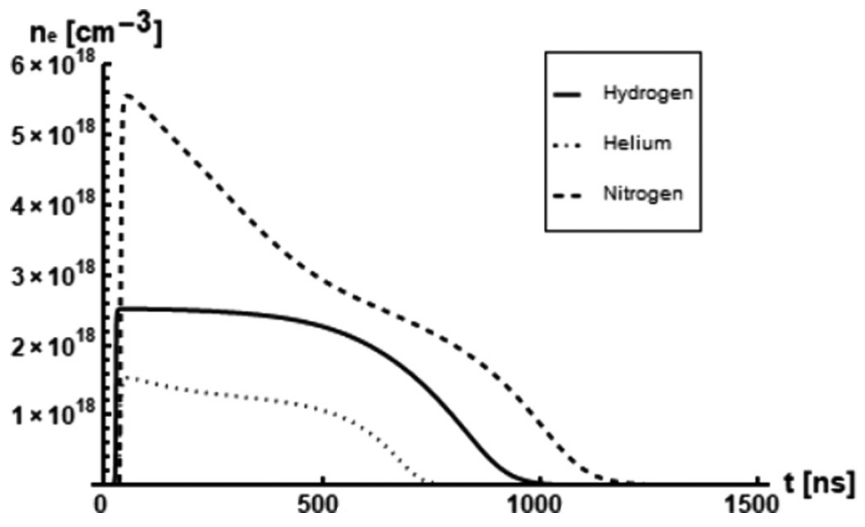

FIG. 19. Simulations referring to the experimental data on Starkbroadening for hydrogen, helium, and nitrogen.

from the helium simulations was $\bar{n}_{e}=1.3 \times 10^{18} \mathrm{~cm}^{-3}$, and finally the one obtained for the nitrogen case was $\bar{n}_{e}=4.1 \times$ $10^{18} \mathrm{~cm}^{-3}$.

\section{Regions of the plasma plumes, the electrodes, and the gas inlets}

In this subsection, we discuss the possible limitations of our diagnostic approach, mainly related to the light emission from the plasma expanding outside the capillary and the plasma eventually formed in the gas inlets' region. Figure 20 shows measurements of plasma density outside the capillary, obtained during a dedicated experiment for characterizing the region of the so-called plasma plumes. The density was obtained by collecting Balmer- $\alpha$ light emitted by the hydrogen plasma with an optical system sending to an imaging spectrometer (SpectraPro275) with a $2400 \mathrm{~g} / \mathrm{mm}$ grating. Therefore, by using an Andor ICCD camera $(255 \times$ 1024 pixels) to acquire the spectrally dispersed plasma light within a time-window of $20 \mathrm{~ns}$ around the instant of maximal density, Stark-broadened spectral lines along the capillary axis provided spatial resolution for the measurement of the electron plasma density [26,27]. According to the scaling for line emission $\propto n_{e}^{2}$ [21], and given the ratio between the electron plasma density at the center of the capillary and that within the plasma plumes $\sim 5$ (see Fig. 20), we conclude that the light emitted by the region outside the capillary is

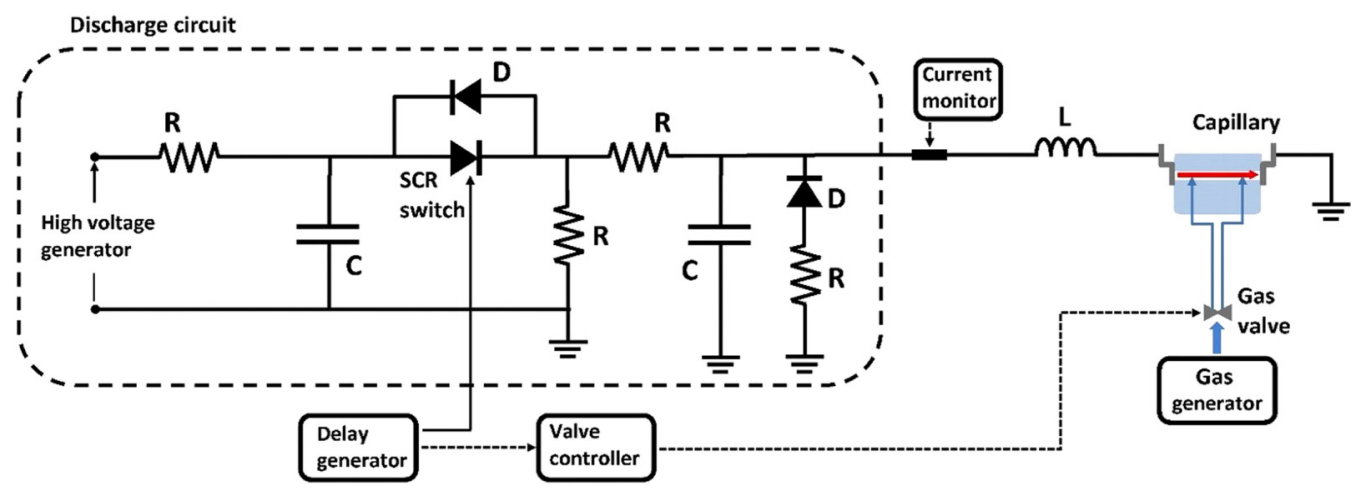

FIG. 18. Schematic of the discharge circuit used for the experiment of electron plasma density measurement. Legend: $R$, resistor; $C$, capacitance; $L$, inductance; $D$, diode; and SCR, silicon-controlled rectifier. 


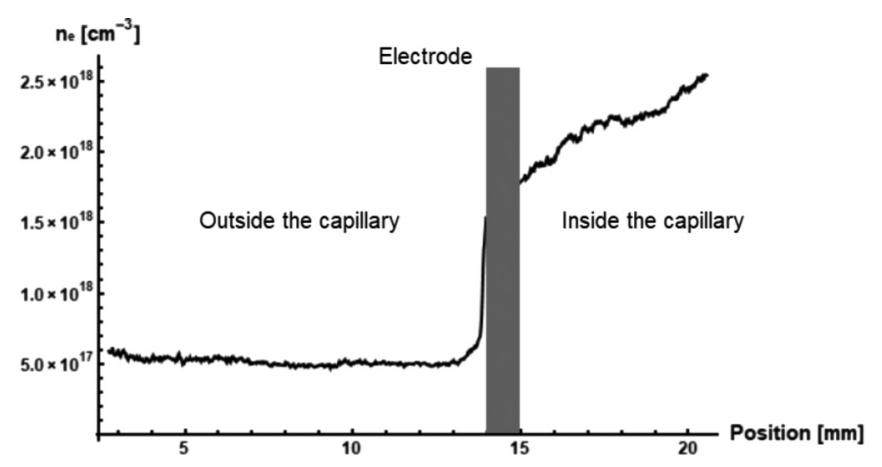

FIG. 20. Characterization of the electron plasma density for the hydrogen plasma outside the capillary.

at least $1 / 25$ of the light emitted by the bulk of the plasma within the capillary walls, even when the volumes of space of the two regions are comparable. The volume shadowed by the electrodes is much smaller than the volume of the bulk plasma $\sim 1 / 15$, and it affects the measurement by preventing that information from that region being recorded during the spectroscopic measurements. Concerning the gas inlets used for the experiment, they were two gas inlets of $0.3 \mathrm{~mm}$ in radius. The timing between the discharge current and the gasinjection was chosen in such a way as to have the discharge only through the gas within the capillary channel, avoiding the risk of damaging the fast electrovalve controlling the gas-flux together with its power supply. Indeed, the electrovalve was open for $3 \mathrm{~ms}$ while the high-voltage was applied after $1.3 \mathrm{~ms}$ in order to synchronize with the gas flowing only within the capillary channel and not within the inlets. It is worth noting that these times strongly depend on the capillary design. The gas ionization was optimal within the capillary only when no arch in the inlets was observed, thus no contribution to plasma light from the inlets' region was recorded during the electron plasma density measurement. Therefore, it is well justified how the numerical model described in Sec. II, considering only the bulk plasma inside the capillary, is in good agreement in terms of electron plasma density with the diagnostic approach recording line emission from the whole capillary apparatus.

\section{CONCLUSIONS}

The present work aimed to introduce a simplified model for the temporal evolution of plasma discharge capillaries, with the chance to study the plasma temperature and all the related quantities such as the ionization degree, the discharge current, and the plasma losses in terms of heat and radiation. Starting from an existing model, large and new improvements have been done in order to include more physical mechanisms, such as heat loss to the capillary walls and radiation losses via radiative recombination and bremsstrahlung. Furthermore, as a further advancement, the possibility of simulating gas mixtures with this model has been created, as well as Debye shielding effects and multi-ionization. An analytic model has been proposed for the calculation of the transverse profile of the temperature and all the related quantities at the thermal equilibrium, which takes into account both heat and radiation losses. This consists in an optimization of the previous model [13], which did not consider the importance of radiation losses. It has been shown that due to the strong nonlinearity of the equilibrium temperature's equation, radiation losses cannot be negligible for plasma parameters commonly adopted in plasma acceleration or focusing experiments. The error committed by neglecting radiation losses can be up to $20 \%$ on quantities of interest such as the radial slope of the magnetic field exploited in plasma lensing experiments, the resonant plasma frequency for wakefield generation in acceleration experiments, as well as the matched spot size of a laser being guided within a plasma channel. Finally, models and diagnostic methods have been described, supported by experimental data, for measuring the electron plasma density by means of atomic and molecular Stark broadening in different gases. Despite the extreme simplicity of the experimental configuration, the results show an optimal agreement with numerical simulations based on the novel model described in Sec. II and with the analytic model for Stark broadening of both spontaneous and induced electric dipoles developed in Sec. VII.
[1] E. Esarey, C. B. Schroeder, and W. P. Leemans, Physics of laserdriven plasma-based electron accelerators, Rev. Mod. Phys. 81, 1229 (2009).

[2] M. Hogan, T. Raubenheimer, A. Seryi, P. Muggli, T. Katsouleas, C. Huang, W. Lu, W. An, K. Marsh, W. Mori et al., Plasma wakefield acceleration experiments at facet, New J. Phys. 12, 055030 (2010).

[3] J. van Tilborg, S. Steinke, C. G. R. Geddes, N. H. Matlis, B. H. Shaw, A. J. Gonsalves, J. V. Huijts, K. Nakamura, J. Daniels, C. B. Schroeder, C. Benedetti, E. Esarey, S. S. Bulanov, N. A. Bobrova, P. V. Sasorov, and W. P. Leemans, Active Plasma Lensing for Relativistic Laser-Plasma-Accelerated Electron Beams, Phys. Rev. Lett. 115, 184802 (2015).

[4] R. Corsini, E. Adli, A. Curcio, S. Curt, S. Döbert, W. Farabolini, D. Gamba, R. Garcia Alia, R. Jones, A. Lagzda et al., First experiments at the clear user facility, in The 9th International Particle Accelerator Conference (IPAC'18), Vancouver, BC, Canada (JACOW, Geneva, Switzerland, 2018), pp. 4066-4069.

[5] R. Pompili, M. P. Anania, M. Bellaveglia, A. Biagioni, S. Bini, F. Bisesto, E. Brentegani, F. Cardelli, G. Castorina, E. Chiadroni, A. Cianchi, O. Coiro, G. Costa, M. Croia, D. Di Giovenale, M. Ferrario, F. Filippi, A. Giribono, V. Lollo, A. Marocchino, M. Marongiu, V. Martinelli, A. Mostacci, D. Pellegrini, L. Piersanti, G. Di Pirro, S. Romeo, A. R. Rossi, J. Scifo, V. Shpakov, A. Stella, C. Vaccarezza, F. Villa, and A. Zigler, Focusing of High-Brightness Electron Beams with Active-Plasma Lenses, Phys. Rev. Lett. 121, 174801 (2018).

[6] R. Pompili, M. Anania, M. Bellaveglia, A. Biagioni, S. Bini, F. Bisesto, E. Brentegani, G. Castorina, E. Chiadroni, A. Cianchi 
et al., Experimental characterization of active plasma lensing for electron beams, Appl. Phys. Lett. 110, 104101 (2017).

[7] C. A. Lindstrøm, E. Adli, G. Boyle, R. Corsini, A. E. Dyson, W. Farabolini, S. M. Hooker, M. Meisel, J. Osterhoff, J.-H. Röckemann, L. Schaper, and K. N. Sjobak, Emittance Preservation in an Aberration-Free Active Plasma Lens, Phys. Rev. Lett. 121, 194801 (2018).

[8] A. Curcio, A. Marocchino, V. Dolci, S. Lupi, and M. Petrarca, Resonant plasma excitation by single-cycle THz pulses, Sci. Rep. 8, 1052 (2018).

[9] M. Ferrario, D. Alesini, M. Anania, M. Artioli, A. Bacci, S. Bartocci, R. Bedogni, M. Bellaveglia, A. Biagioni, F. Bisesto etal., Eupraxia@sparc_lab design study towards a compact fel facility at lnf, Nucl. Instrum. Methods Phys. Res. Sec. A 909, 134 (2018).

[10] A. Curcio, M. Petrarca, D. Giulietti, and M. Ferrario, Numerical and analytical models to study the laser-driven plasma perturbation in a dielectric gas-filled capillary waveguide, Opt. Lett. 41, 4233 (2016).

[11] A. Curcio and M. Petrarca, Diagnosing plasmas with wideband terahertz pulses, Opt. Lett. 44, 1011 (2019).

[12] A. Curcio, D. Giulietti, and M. Petrarca, Tuning of betatron radiation in laser-plasma accelerators via multimodal laser propagation through capillary waveguides, Phys. Plasmas 24, 023104 (2017).

[13] N. A. Bobrova, A. A. Esaulov, J.-I. Sakai, P. V. Sasorov, D. J. Spence, A. Butler, S. M. Hooker, and S. V. Bulanov, Simulations of a hydrogen-filled capillary discharge waveguide, Phys. Rev. E 65, 016407 (2001).

[14] J.-H. Röckemann, L. Schaper, S. K. Barber, N. A. Bobrova, G. Boyle, S. Bulanov, N. Delbos, K. Floettmann, G. Kube, W. Lauth, W. P. Leemans, V. Libov, A. R. Maier, M. Meisel, P. Messner, P. V. Sasorov, C. B. Schroeder, J. van Tilborg, S. Wesch, and J. Osterhoff, Direct measurement of focusing fields in active plasma lenses, Phys. Rev. Accel. Beams 21, 122801 (2018).

[15] M. Anania, E. Chiadroni, A. Cianchi, D. Di Giovenale, M. Ferrario, F. Flora, G. Gallerano, A. Ghigo, A. Marocchino, F. Massimo et al., Design of a plasma discharge circuit for particle wakefield acceleration, Nucl. Instrum. Methods Phys. Res. Sec. A 740, 193 (2014).
[16] M. Anania, A. Biagioni, E. Chiadroni, A. Cianchi, M. Croia, A. Curcio, D. Di Giovenale, G. Di Pirro, F. Filippi, A. Ghigo et al., Plasma production for electron acceleration by resonant plasma wave, Nucl. Instrum. Methods Phys. Res. Sec. A 829. 254 (2016).

[17] F. Bisesto, M. Anania, M. Bellaveglia, E. Chiadroni, A. Cianchi, G. Costa, A. Curcio, D. D. Giovenale, G. D. Pirro, M. Ferrario, F. Filippi, A. Gallo, A. Marocchino, R. Pompili, A. Zigler, and C. Vaccarezza, The flame laser at sparc_lab, Nucl. Instrum. Methods Phys. Res. Sec. A 909, 452 (2018), 3rd European Advanced Accelerator Concepts Workshop (EAAC2017).

[18] S. Eliezer, The Interaction of High-power Lasers with Plasmas (CRC, Boca Raton, FL, 2002).

[19] D. Book, and J. D. Huba, NRL plasma formulary No. NRL/PU6790-02-450, Naval Research Lab, Washington DC, Plasma Physics Division, 2002.

[20] L. Steinhauer and W. Kimura, Quasistatic capillary discharge plasma model, Phys. Rev. Spec. Top.-Accel. Beams 9, 081301 (2006).

[21] C. Alessandro and D. Giulietti, Laser-plasma Acceleration and Secondary em Radiation (Aracne, Rome, 2019).

[22] H. Bethe, Zur theorie des durchgangs schneller korpuskularstrahlen durch materie, Ann. Phys. 397, 325 (1930).

[23] H. Griem, Spectral Line Broadening by Plasmas (Elsevier, Amsterdam, 2012).

[24] M. A. Gigosos, M. Á. González, and V. Cardeñoso, Computer simulated Balmer-alpha,-beta and-gamma stark line profiles for non-equilibrium plasmas diagnostics, Spectrochim. Acta, Part B 58, 1489 (2003).

[25] H. R. Griem, J. Halenka, and W. Olchawa, Comparison of hydrogen Balmer-alpha stark profiles measured at high electron densities with theoretical results, J. Phys. B 38, 975 (2005).

[26] A. J. Gonsalves, T. P. Rowlands-Rees, B. H. P. Broks, J. J. A. M. van der Mullen, and S. M. Hooker, Transverse Interferometry of a Hydrogen-Filled Capillary Discharge Waveguide, Phys. Rev. Lett. 98, 025002 (2007).

[27] A. Biagioni, D. Alesini, M. Anania, M. Bellaveglia, S. Bini, F. Bisesto, E. Brentegani, E. Chiadroni, A. Cianchi, O. Coiro et al., Temperature analysis in the shock waves regime for gas-filled plasma capillaries in plasma-based accelerators, J. Instrum. 14, C03002 (2019). 